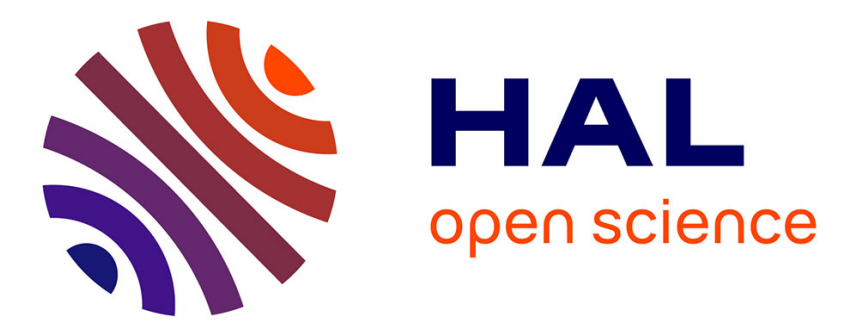

\title{
Distributed Enhanced Inter-Cell Interference Coordination (eICIC) in LTE-Advanced HetNets: A Potential Game Approach
}

\author{
Ye Liu, Chung Shue Chen, Chi Wan Sung
}

\section{- To cite this version:}

Ye Liu, Chung Shue Chen, Chi Wan Sung. Distributed Enhanced Inter-Cell Interference Coordination (eICIC) in LTE-Advanced HetNets: A Potential Game Approach. IEEE VTC-Spring, Workshop on Intelligent Design and Performance Evaluation of LTE-Advanced Networks, May 2015, Glasgow, United Kingdom. hal-01471135

\author{
HAL Id: hal-01471135 \\ https://hal.inria.fr/hal-01471135
}

Submitted on 19 Feb 2017

HAL is a multi-disciplinary open access archive for the deposit and dissemination of scientific research documents, whether they are published or not. The documents may come from teaching and research institutions in France or abroad, or from public or private research centers.
L'archive ouverte pluridisciplinaire $\mathbf{H A L}$, est destinée au dépôt et à la diffusion de documents scientifiques de niveau recherche, publiés ou non, émanant des établissements d'enseignement et de recherche français ou étrangers, des laboratoires publics ou privés. 


\section{Distributed Enhanced Inter-Cell Interference Coordination (eICIC) in LTE-Advanced HetNets: A Potential Game Approach}

\author{
Ye Liu and Chung Shue Chen \\ Alcatel-Lucent Bell-Labs \\ Centre de Villarceaux, 91620 Nozay, France \\ Email: \{ye.liu1, cs.chen\}@alcatel-lucent.com
}

\author{
Chi Wan Sung \\ City University of Hong Kong \\ Tat Chee Avenue, Kowloon, Hong Kong SAR \\ Email: albert.sung@ cityu.edu.hk
}

\begin{abstract}
In this paper we propose a distributed algorithm for jointly optimizing almost blank subframe (ABS) and cell selection bias (CSB) patterns in Long Term Evolution-Advanced (LTE-A) heterogeneous networks (HetNets). We formulate the optimization problem as an exact potential game, where a Nash equilibrium point is guaranteed to be achieved within finite number of plays. Through simulations, we are able to demonstrate the fast convergence of the algorithm, an increase in average user rate, and a tremendous improvement on the service fairness of the users.

Index Terms-LTE/LTE-A, enhanced inter-cell interference coordination (eICIC), almost blank subframe (ABS), cell selection bias (CSB), heterogeneous networks (HetNets), distributed optimization, potential game.
\end{abstract}

\section{INTRODUCTION}

Heterogeneous cellular networks become an attractive solution to the exponentially growing data traffic of today's mobile networks. In frequency reuse-1 settings, however, the strong interference from the macrocells can severely degrade the reception quality of the users attached to the picocells. Besides, users who are located near and can attach to a small cell may be attached to a macrocell because the reference signal received power (RSRP) from the macrocell can be much larger than that from the picocell, which may lead to underutilization of the picocell. To tackle the aforementioned challenges in heterogeneous networks (HetNets) and fully utilize the power of picocells, 3rd Generation Partnership Project (3GPP) proposes enhanced inter-cell interference coordination (eICIC), where almost blank subframe (ABS) and cell selection bias (CSB) patterns can be employed to tune the network and optimize the radio resource utilization [1].

Several algorithms of optimizing the ABS and/or CSB settings have been proposed. Tall et al. propose an algorithm that optimizes ABS and CSB separately in [2], where the ABS patterns are simplified as fractional numbers. A centralized algorithm that jointly optimizes ABS and CSB patterns is proposed by Deb et al. in [3], where the interfering macrocells of a picocell offer ABSs on the same subframes. Pang et al. discuss a distributed algorithm to determine the number of ABSs in [4], yet they do not consider CSB optimization. Simsek et al. propose a learning algorithm that dynamically optimize CSB in frequency domain in [5] and extend the idea to optimize CSB in both time and frequency domain in [6].
In this paper, we provide a distributed algorithm that jointly optimizes the ABS and CSB patterns in HetNets. We formulate the problem as an exact potential game, where the cells in the network are the players and the ABS and/or CSB patterns are the strategies. A decentralized eICIC algorithm is therefore derived, based on the given potential game. We show that such an approach can be used to maximize various objectives, such as the sum of users' throughput and the sum of logarithmic utilities of users' throughput. Through simulation studies, we show that the algorithm can quickly converge, and a 50\% improvement on average user throughput can be achieved by optimizing the ABS and CSB patterns. Meanwhile, the service fairness among users can also be greatly improved.

The rest of the paper is organized as follows: Section II presents the system model of Long Term Evolution-Advanced (LTE-A) HetNets and the joint ABS and CSB optimization problem. Section III derives the exact potential game formulation. Section IV discusses the required information to perform the optimization. Section $\mathrm{V}$ shows the simulation results and Section VI gives the conclusion.

\section{System Model}

Consider a LTE-A HetNet where macrocells and picocells coexist. Let $\mathcal{M}$ be the set of all macrocells and $\mathcal{P}$ be the set of all picocells. In the sequel a station may either be referred to as a macrocell or a picocell. Let $\mathcal{U}_{i}$ be the set of users associated with station $i$, where $i \in \mathcal{M} \cup \mathcal{P}$. The $|\mathcal{M}||\mathcal{P}|$ by- 1 vector $\gamma$ specifies the CSB values of all macrocells and picocells, where $\gamma_{i}$, the CSB value of station $i$, belongs to a pre-defined set $\mathcal{C}$. Let $P_{\mathrm{Rx}, u}^{i}$ be the RSRP of user $u$ from station $i$, where $i \in \mathcal{M} \cup \mathcal{P}$ and the received power depends on the transmission power of the station, the distance between the station and the UE, and the shadowing loss. User $u$ will be attached to the following station

$$
g(u, \gamma) \triangleq \arg \max _{i}\left(P_{\mathrm{Rx}, u}^{i}+\gamma_{i}\right) .
$$

Assume there are $T$ subframes in the time domain and $F$ resource blocks $(\mathrm{RBs})$ in the frequency domain. Each subframe and RB pair forms a physical resource block (PRB), where a subframe has a duration of $1 \mathrm{~ms}$ and a RB has a bandwidth of $180 \mathrm{kHz}$. Let $B:=T \cdot F$ be the total number of PRBs available at each station. All macrocells transmit at a fixed power level 
on PRBs which correspond to non-ABSs, and all picocells transmit at a fixed power level on all PRBs. All subframes used by all stations are assumed to be synchronized.

The achieved rate of user $u$ at PRB $b$ is denoted as $r_{u, b}$, where $b \in[1, B]$. Due to the small-scale fading and potentially different ABS patterns of the interfering macrocells, a user can get different throughput on different PRBs. Let $\boldsymbol{\alpha}_{m}$ be the $T$ by-1 binary vector specifying the ABS pattern of macrocell $m$, where $\boldsymbol{\alpha}_{m, i}=0$ means the $i$-th subframe of macrocell $m$ is an ABS and $\boldsymbol{\alpha}_{m, j}=1$ means the $j$-th subframe of macrocell $m$ is a non-ABS, respectively. The Signal-to-Noiseplus-Interference Ratio (SINR) of a user $u$ associated with an macrocell $m$ on PRB $b$ is given as:

$$
\operatorname{SINR}_{u, b}^{m}=\frac{h_{u, b}^{m} \cdot P_{\mathrm{Rx}, u}^{m} \cdot \boldsymbol{\alpha}_{m, \tau(b)}}{P_{\mathrm{Int}, u, b}^{\mathcal{M} \backslash\{m\}}+P_{\mathrm{Int}, u, b}^{\mathcal{P}}+N_{0}},
$$

where $h_{u, b}^{m}$ is the fading gain on PRB $b$ from macrocell $m$ to user $u, \tau(b)$ returns the index of subframe of PRB $b, P_{\text {Int }, b}^{\mathcal{M} \backslash\{m\}}$ is the interference from macrocells in $\mathcal{M} \backslash\{m\}$ at PRB $b, P_{\text {Int } b}^{\mathcal{P}}$ is the interference from picocells in $\mathcal{P}$ at $\mathrm{PRB} b$, and $N_{0}$ is the Additive-White-Gaussian Noise (AWGN). Note that $P_{\mathrm{Int}, u, b}^{\mathcal{M} \backslash\{m\}}$ depends on the ABS patterns of the macrocells in $\mathcal{M} \backslash\{m\}$ and the large and small scale fading gains of the channels from the macrocells in $\mathcal{M} \backslash\{m\}$ to user $u$. Also, note that $P_{\text {Int }, u, b}^{\mathcal{P}}$ depends on the large and small scale fading gains of the channels from the picocells in $\mathcal{P}$ to user $u$. The SINR of a user $u$ associated with picocell $p \in \mathcal{P}$ at PRB $b$ is given as:

$$
\operatorname{SINR}_{u, b}^{p}=\frac{h_{u, b}^{p} \cdot P_{\mathrm{Rx}, u}^{p}}{P_{\mathrm{Int}, u, b}^{\mathcal{M}}+P_{\mathrm{Int}, u, b}^{\mathcal{P} \backslash\{p\}}+N_{0}},
$$

where $h_{u, b}^{p}$ is the channel gain from picocell $p$ to user $u$ at PRB $b, P_{\text {Int }, u, b}^{\mathcal{M}}$ is the interference from macrocells in $\mathcal{M}$, and $P_{\text {Int }, u}^{\mathcal{P} \backslash\{p\}}$ is the interference from picocells in $\mathcal{P} \backslash\{p\}$.

Given the above SINR definitions and user association, the throughput at PRB $b$ of a user $u$, namely $r_{u, b}$, can be calculated by Shannon's capacity formula.

Assume $x_{u, b}$ is a binary value specifying whether user $u$ occupies the $b$-th PRB of its serving station, where $x_{u, b}=1$ and $x_{u, b}=0$ mean user $u$ occupies and does not occupy the $b$-th $\mathrm{PRB}$, respectively. Let $w_{u}$ be a nonnegative weighting factor of UE $u$. The optimization problem can be stated as:

$$
\begin{array}{cc}
\operatorname{maximize} & \sum_{i \in \mathcal{M} \cup \mathcal{P}} \sum_{u \in \mathcal{U}_{i}} w_{u} \cdot \ln \sum_{b=1}^{B} x_{u, b} \cdot r_{u, b}, \\
\text { subject to } & \sum_{u \in \mathcal{U}_{m}} x_{u, b}=\boldsymbol{\alpha}_{m, \tau(b)}, \forall m \in \mathcal{M}, \\
b \in[1, B], \boldsymbol{\alpha}_{m} \in \mathcal{A}, \\
\sum_{u \in \mathcal{U}_{p}} x_{u, b}=1, \forall p \in \mathcal{P}, b \in[1, B], \\
x_{u, b} \in\{0,1\}, \forall u \in \mathcal{U}, b \in[1, B], \\
\gamma(i) \in \mathcal{C}, \forall i \in \mathcal{M} \cup \mathcal{P},
\end{array}
$$

where $\mathcal{A}$ is the set of vectors from which macrocells can select their ABS pattern. Note that we choose to maximize the proportional fair (PF) objective, i.e., sum of $\log$ of user rates, because it strikes a balance between network throughput and user fairness (e.g., see [7]).

\section{Distributed Solution Using Potential Game}

In this section, we aim at developing a distributed solution to the problem described in Section II by formulating an exact potential game.

\section{A. Exact Potential Game Formulation}

In an exact potential game, there exists a potential function such that the change in the potential function due to the change of a player's strategy equals to the change of the utility of that player. A Nash equilibrium can be reached within a finite number of plays if a player is randomly chosen to update its strategy so that the player's utility is maximized in each step [8]. In [9], an exact potential game is formulated to optimize parameters in LTE, where the users in the system are the players. In the following, we show that the same idea can be used in the current context where a potential game is formulated by using the stations as the players. After all, it is not practical to let the users to control the cellular network.

Let the stations be the players, so that $\mathcal{M} \cup \mathcal{P}$ defines the set of players. In the sequel we use station and player interchangeably. Each player $i$ chooses a strategy $s_{i}$ from a set of strategies $\mathcal{S}_{i}$. The underlying assumption is that ABS is allowed in macrocells but not in picocells, and all stations can set their CSB values according to $\mathcal{C}$. Let vector $\mathbf{s}$ specify the strategies of all players, where $\mathbf{s}(i)$ gives the strategy of station $i$. Define the strategies selected by all players except player $i$ as

$$
\mathbf{s}_{-i} \triangleq\left(s_{1}, \ldots, s_{i-1}, s_{i+1}, \ldots, s_{|\mathcal{M}|+|\mathcal{P}|}\right),
$$

and define

$$
\left(s_{i}^{\prime}, \mathbf{s}_{-i}\right) \triangleq\left(s_{1}, \ldots, s_{i-1}, s_{i}^{\prime}, s_{i+1}, \ldots, s_{|\mathcal{M}|+|\mathcal{P}|}\right)
$$

so that player $i$ adopts strategy $s_{i}^{\prime}$ and other players' strategies are specified by $\mathbf{s}_{-i}$.

Let $\mathcal{N}_{i} \triangleq\{i\} \cup \mathcal{N}_{i}^{\text {Int }} \cup \mathcal{N}_{i}^{\text {Att }}$, where $i \in \mathcal{M} \cup \mathcal{P}, \mathcal{N}_{i}^{\text {Int }}$ is the set of stations whose attached users can be interfered by the transmissions of station $i, \mathcal{N}_{i}^{\text {Att }}$ is the set of stations whose user attachment can be affected by the change of CSB value of station $i$. Let the utility of station $i \in \mathcal{M} \cup \mathcal{P}$ be

$$
U_{i}(\mathbf{s}) \triangleq \sum_{u \in \mathcal{U}_{i}} w_{u} \cdot \ln \sum_{b=1}^{B} x_{u, b} \cdot r_{u, b}
$$

where $x_{u, b}$ is obtained by some scheduling scheme given s so that the constraint in (3), (4) and (5) are satisfied. The utility of the whole system is

$$
U(\mathbf{s})=\sum_{i \in \mathcal{M} \cup \mathcal{P}} U_{i}(\mathbf{s}),
$$

Let the payoff function of player $i$ be

$$
V_{i}(\mathbf{s}) \triangleq \sum_{j \in \mathcal{N}_{i}} U_{j}(\mathbf{s}) .
$$


We have,

$$
\begin{array}{cc} 
& U\left(s_{i}^{\prime}, \mathbf{s}_{-1}\right)-U(\mathbf{s}) \\
= & \sum_{j \in \mathcal{M} \cup \mathcal{P}} U_{j}\left(s_{i}^{\prime}, \mathbf{s}_{-1}\right)-U_{j}(\mathbf{s}) \\
= & \sum_{j \in \mathcal{N}_{i}}\left(U_{j}\left(s_{i}^{\prime}, \mathbf{s}_{-1}\right)-U_{j}(\mathbf{s})\right)+ \\
= & \sum_{j \in \mathcal{M} \cup \mathcal{P} \backslash \mathcal{N}_{i}}\left(U_{j}\left(s_{i}^{\prime}, \mathbf{s}_{-1}\right)-U_{j}(\mathbf{s})\right) \\
= & \sum_{j \in \mathcal{N}_{i}}\left(U_{j}\left(s_{i}^{\prime}, \mathbf{s}_{-1}\right)-U_{j}(\mathbf{s})\right) \\
= & V_{i}\left(\left(s_{i}^{\prime}, \mathbf{s}_{-i}\right)\right)-V_{i}(\mathbf{s}),
\end{array}
$$

where (9) follows from the fact that the change of player $i$ 's strategy will only affect the utilities of players in $\mathcal{N}_{i}$. Eqn. (10) shows that $U(\cdot)$ is a potential function of the game $(\mathcal{M} \cup$ $\left.\mathcal{P},\left(\mathcal{S}_{i}, i \in \mathcal{M} \cup \mathcal{P}\right),\left(V_{i}, i \in \mathcal{M} \cup \mathcal{P}\right)\right)$.

Note that the utility function in (7) can be modified to other functions without affecting the validity of the potential game formulation, as long as the utility function depends on the users who are attached to a single station. For example, it can be $\sum_{u \in \mathcal{U}_{i}} w_{u} \cdot \sum_{b=1}^{B} x_{u, b} \cdot r_{u, b}$, or in other words the weighted sum of user rates [10].

The procedure of game play is given as follows:

1) Initialize the ABS and CSB patterns of all macrocells and picocells.

2) Randomly select a player $i \in \mathcal{M} \cup \mathcal{P}$. The player then searches through all strategies in $\mathcal{S}_{i}$ and chooses a strategy so that $V_{i}$ is maximized.

a) $\mathrm{ABS}$ and $\mathrm{CSB}$ optimization: $\mathcal{S}_{i}:=\mathcal{A} \times \mathcal{C}$ for $i \in$ $\mathcal{M} . \mathcal{S}_{i}:=\mathcal{C}$ for $i \in \mathcal{P}$.

b) ABS optimization: $\mathcal{S}_{i}:=\mathcal{A}$ for $i \in \mathcal{M} . \mathcal{S}_{i}:=\{\overrightarrow{1}\}$ for $i \in \mathcal{P}$, where $\overrightarrow{1}$ means the vector of all $1 \mathrm{~s}$.

c) CSB optimization: $\mathcal{S}_{i}:=\mathcal{C}$ for $i \in \mathcal{M} \cup \mathcal{P}$.

3) Repeat step 2 until some stopping criterion is met.

The utility of a station is dependent not only on its ABS and/or CSB patterns but also on the way it schedules the users. In the following we present the PF scheduler used in later simulation studies to meet the objective in (2).

\section{B. PF Scheduler}

In this paper, we consider the following PF scheduler [11]: For station $i$, the $b$-th $\mathrm{PRB}$ at subframe $t$, given that the subframe is not an ABS, will be allocated to the following user

$$
\widehat{u}_{b} \triangleq \arg \max _{u \in \mathcal{U}_{i}} \frac{r_{u, b}}{\bar{r}_{u}(t)},
$$

where $b \in[1, B], t=\tau(b), \bar{r}_{u}(t)$ gives the long-term average throughput of user $u$ in subframe $t$ and it is calculated as

$$
\bar{r}_{u}(t)=\left(1-\frac{1}{t_{c}}\right) \bar{r}_{u}(t-1)+\frac{1}{t_{c}} \Sigma_{\{b \mid \tau(b)=t\}} r_{u, b} \cdot I\left\{\widehat{u}_{b}=u\right\} .
$$

In the above equation, $t_{c}$ is the time window which is a design parameter and $I\{\cdot\}$ is the indicator function.

\section{INFORMATION EXCHANGE REQUIREMENT}

It is important to know the information that is required to play the potential game, as it is directly related to implementation complexity.

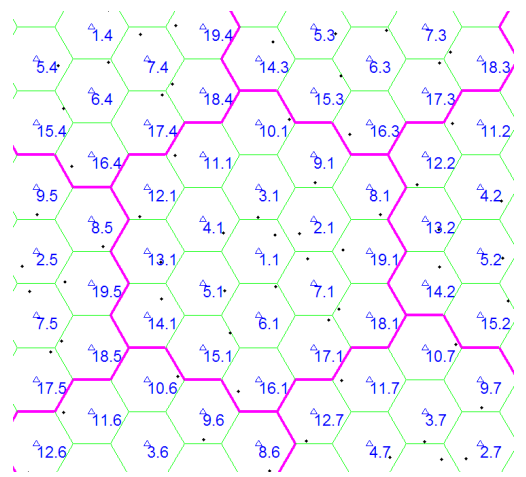

Fig. 1. A randomly generated hexagonal HetNet layout. The triangles represent macrocells and the dots represent picocells. The number 4.1 near one of the triangles indicates that it is an macrocell located in the first (also the center) cluster with reference number 4 . The users are not plotted for clean presentation.

For the selected player $i$, the required information is:

1) Users who attach to player $i$ when player $i$ adopts one of the CSB values in $\mathcal{C}$.

2) Utilities of stations in $\mathcal{N}_{i} \backslash\{i\}$, i.e., $\left\{U_{j} \mid j \in \mathcal{N}_{i} \backslash\{i\}\right\}$, when player $i$ adopts different strategies in $\mathcal{S}_{i}$.

For stations in $\mathcal{N}_{i} \backslash\{i\}$ :

1) They need to know which users will attach to them if station $i$ uses one of the CSB values in $\mathcal{C}$.

2) They need to know the interference power from station $i$ to the users attached to stations in $\mathcal{N}_{i} \backslash\{i\}$. In case station $i$ is an macrocell, the interference power is dependent on the ABS patterns that station $i$ may adopt.

\section{NUMERICAL STUDIES}

Fig. 1 shows a randomly generated hexagonal HetNet consisting of seven clusters of macrocells, where the clusters' borders are marked with bolded lines. For the center cluster, the macrocells are located at the center of the hexagons and one picocell is randomly placed within each hexagon so that the distance between the picocell and its closest macrocell follows the triangular distribution. 14 users are randomly located within 40 meters of each picocell in the center cluster, and another 6 users are randomly located within each hexagon in the center cluster. The six surrounding clusters of the center cluster are copies of the center clusters. The sets $\mathcal{M}$ and $\mathcal{P}$ contain macrocells and the picocells, respectively, in the center cluster. The users are assumed to be static and the buffers of the stations are assumed to be fully loaded. All users are equally weighted, i.e., $w_{u}=1$ for all $u$. Each PRB experiences independent Rayleigh fading with variance 1. Shadowing is correlated, and the correlation is achieved by generating a common shadowing value and divide the sum of the common shadowing value and the individual shadowing value by $\sqrt{2}$. Other parameters can be found in Table I.

Fig. 2 shows the possible ABS patterns of an macrocell. The characteristic of the set of ABS patterns in Fig. 2 is that subframes which are ABSs in an ABS pattern with lower ABS ratio are also ABSs in an $\mathrm{ABS}$ pattern with higher $\mathrm{ABS}$ ratio. 


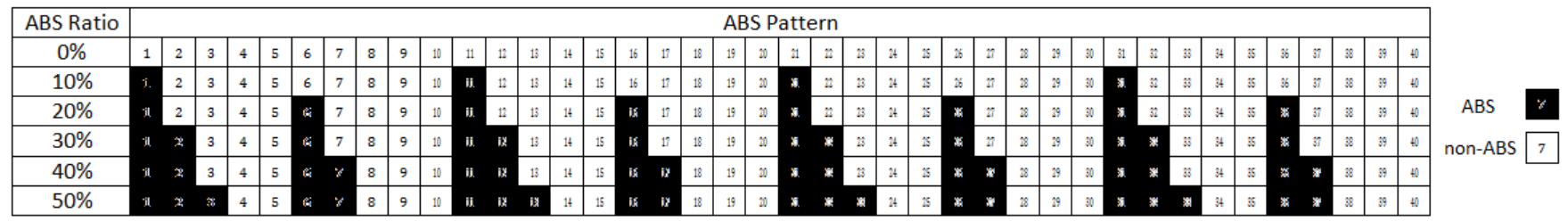

Fig. 2. A set of ABS patterns, where the filled boxes indicate ABSs. Subframes that are set as ABSs in a pattern with a lower ABS ratio are also set as ABSs in a pattern with a higher ABS ratio.

TABLE I

PARAmeters For Generating HetNet Topologies

\begin{tabular}{|c|c|}
\hline Parameter & Value \\
\hline Inter-macrocell distance & $1000 \mathrm{~m}$ \\
\hline Minimum distance from macro to user & $35 \mathrm{~m}$ \\
\hline Minimum distance from pico to user & $10 \mathrm{~m}$ \\
\hline Minimum distance from macro to pico & $75 \mathrm{~m}$ \\
\hline Antenna per site & Omnidirectional $\times 1$ \\
\hline Macrocell power & $10 \mathrm{~W}$ \\
\hline Picocell Power & $-174 \mathrm{dBm} / \mathrm{Hz}$ \\
\hline Noise density & $9 \mathrm{~dB}$ \\
\hline Noise figure & $180 \mathrm{kHz}$ \\
\hline Bandwidth per RB & $10 \mathrm{~dB}$ \\
\hline CSB values & $0 \mathrm{~dB}, 5 \mathrm{~dB}, 10 \mathrm{~dB}, 15 \mathrm{~dB}$ \\
\hline Log-normal shadowing SD & $128.1+37.6 \log _{10} d, d$ in $\mathrm{km}$ \\
\hline Path loss from macrocell to user & $140.7+36.7 \log _{10} d, d$ in $\mathrm{km}$ \\
\hline Path loss from picocell to user
\end{tabular}

This kind of design may allow the surrounding macrocells of a picocell to create subframes that are almost interference free even if the macrocells uses different levels of ABS ratios. On the other hand, the potential game formulation allows a designer to use any kind of ABS and CSB patterns, as the strategy set of a player can be easily modified.

Next we specify the set $\mathcal{N}_{i}$ for $i \in \mathcal{M} \cup \mathcal{P}$. We assume that the transmission of station $i$ will create interference to the users who are located within the same hexagon of station $i$ and the six surrounding hexagons. Also, we assume that a user can only be associated to stations in the same hexagon and its six surrounding hexagons. Therefore, $\mathcal{N}_{i}^{\text {Int }}$ and $\mathcal{N}_{i}^{\text {Att }}$ include stations located within the same hexagon of $i$ and the twelve surrounding hexagons. For example, $\mathcal{N}_{1.1}$ includes all stations in the center cluster in Fig. 1.

Fig. 3 plots the change of global utilities (obtained by (8)) using different scheduling schemes as the number of potential game plays increases. For the PF scheduler, three window sizes are evaluated, namely $t_{c}=5, t_{c}=20$, and $t_{c}=40$. The plot is obtained by averaging 186 randomly generated network topologies. The global utilities increase as the game is played more times because in each round a player uses a best response strategy, i.e., select a strategy that maximizes its payoff function. Also, the algorithm converges in around 150 iterations. We can also observe that the global utility does not improve much if only the CSB values are optimized, and much of the improvement achieved by ABS and CSB optimization can also be achieved by ABS optimization along. We believe this is reasonable because most of the users are located near the picocell in each hexagon so that they receive fairly good

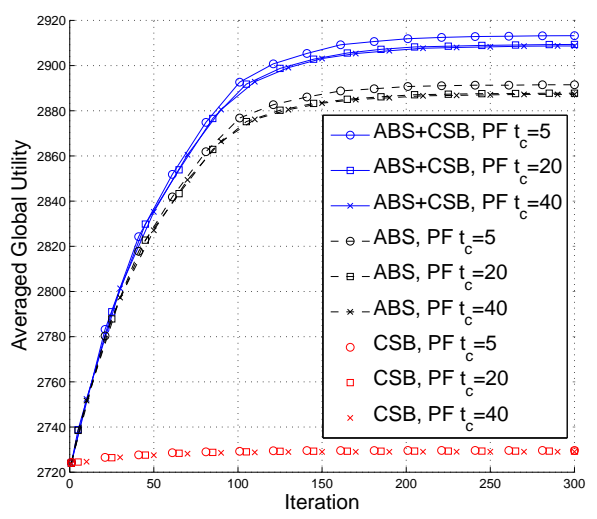

Fig. 3. Averaged global utility against the number of times the potential game is being played using various scheduling schemes.

service from the picocell, and each picocell has plenty PRBs to serve its associated users.

Fig. 4 plots the empirical cumulative distribution functions (CDFs) of the rates of the users averaged over their number of allocated PRBs after 300 iterations of game play, where both $\mathrm{ABS}$ and CSB patterns are optimized. We can observe that the users' rates per PRB are greatly increased due to the ABS and CSB optimization. Specifically, the median rate after ABS and CSB optimization is increased by roughly $60 \%$ compared to the case where no optimization is performed. Fig. 5 and Fig. 6 plot the CDFs of the averaged rates per PRB when ABS optimization is performed and when CSB optimization is performed, respectively. We can see from Fig. 5 that the improvement due to ABS optimization is similar to the improvement due to ABS and CSB optimization. Also, we can observe from Fig. 6 that by performing CSB optimization along, little improvement can be obtained. These observations coincide with the observations from Fig. 3.

Table II summarizes some key performance indicators of various optimization scenarios. We can observe that the ABS and CSB optimization gives about 10\% improvement on the mean achieved user rate compared to the case where no optimization is performed, where a user's achieved rate is calculated by adding the capacity of each PRB that is allocated to that user. Also, the Jain's fairness indices on users' throughput when ABS and CSB optimization is performed are greatly improved compared to other cases. Moreover, we can see that the PF scheduler with time window being 5 has the 


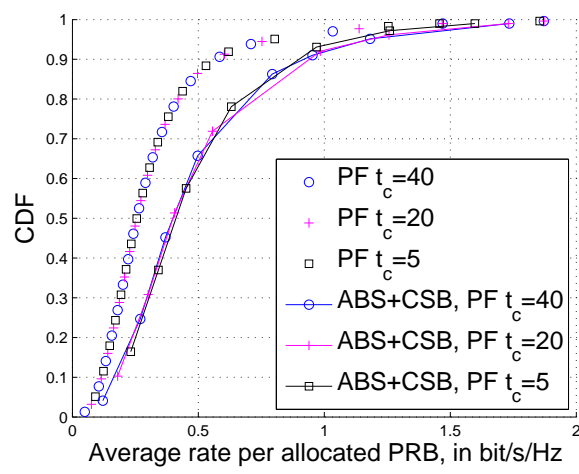

Fig. 4. CDF plot of throughput of users who are located in the center cluster in all randomly generated topologies when joint optimization (i.e., ABS and CSB joint optimization) is performed.

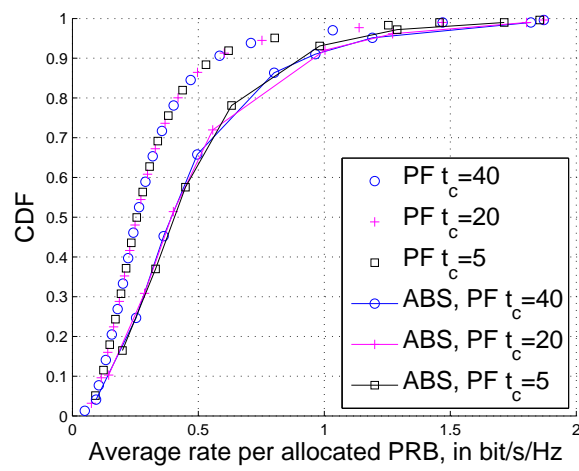

Fig. 5. CDF plot of throughput of users who are located in the center cluster in all randomly generated topologies when ABS optimization is performed.

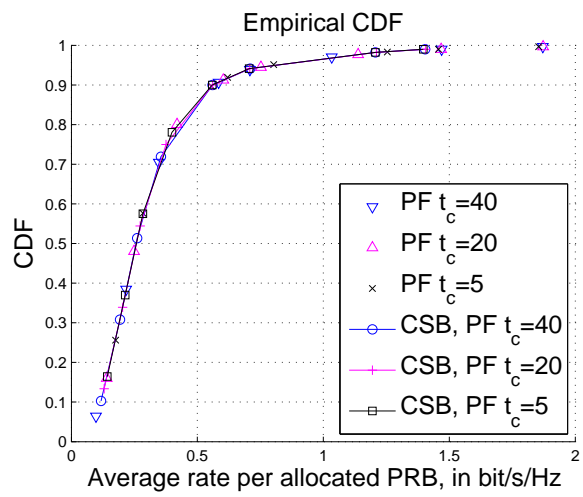

Fig. 6. CDF plot of throughput of users who are located in the center cluster in all randomly generated topologies when CSB optimization is performed.

largest fairness index and the least mean achieved user rate in all optimization scenarios.

\section{CONCLUSION}

We propose a distributed eICIC optimization algorithm based on potential game formulation. We show that such formulation can be used to optimize different objectives, e.g., maximizing proportional fair and maximizing sum of users throughput. Simulation studies show that the optimization
TABLE II

FAIRness Indices AND AVERAge ACHIEVEd User RATE

\begin{tabular}{|c|c|c|c|}
\hline Optimization & Scheduler & Jain's Index & $\begin{array}{c}\text { Mean UE Rate } \\
(\mathrm{bit} / \mathrm{s} / \mathrm{Hz})\end{array}$ \\
\hline \multirow{4}{*}{ ABS+CSB } & $\mathrm{PF} t_{c}=5$ & 0.8263 & 186.89 \\
\cline { 2 - 4 } & $\mathrm{PF} t_{c}=20$ & 0.8276 & 192.93 \\
\cline { 2 - 4 } & $\mathrm{PF} t_{c}=40$ & 0.8281 & 193.66 \\
\cline { 2 - 4 } & $\mathrm{RR}$ & 0.7904 & 154.09 \\
\hline \multirow{5}{*}{$\mathrm{ABS}$} & $\mathrm{PF} t_{c}=5$ & 0.7799 & 183.25 \\
\cline { 2 - 4 } & $\mathrm{PF} t_{c}=20$ & 0.7798 & 189.49 \\
\cline { 2 - 4 } & $\mathrm{PF} t_{c}=40$ & 0.7796 & 190.30 \\
\cline { 2 - 4 } & $\mathrm{RR}$ & 0.7152 & 149.34 \\
\hline \multirow{5}{*}{$\mathrm{CSB}$} & $\mathrm{PF} t_{c}=5$ & 0.7213 & 167.33 \\
\cline { 2 - 4 } & $\mathrm{PF} t_{c}=20$ & 0.7246 & 171.27 \\
\cline { 2 - 4 } & $\mathrm{PF} t_{c}=40$ & 0.7250 & 171.77 \\
\cline { 2 - 4 } & $\mathrm{RR}$ & 0.6517 & 124.93 \\
\hline \multirow{5}{*}{ No optimization } & $\mathrm{PF} t_{c}=5$ & 0.7002 & 167.86 \\
\cline { 2 - 4 } & $\mathrm{PF} t_{c}=20$ & 0.7057 & 171.79 \\
\cline { 2 - 4 } & $\mathrm{PF} t_{c}=40$ & 0.7063 & 172.27 \\
\cline { 2 - 4 } & $\mathrm{RR}$ & 0.6343 & 125.41 \\
\hline
\end{tabular}

algorithm can lead to $10 \%$ increase in average achieved user rate while at the same time greatly increase the service fairness of the network.

\section{ACKNOWLEDGEMENT}

This work was supported by ANR project IDEFIX under grant number ANR-13-INFR-0006 and in part by a grant from the Research Grants Council of the Hong Kong Special Administrative Region, China, under Project CityU 121713. A part of the work was carried out at LINCS (www.lincs.fr).

\section{REFERENCES}

[1] D. Lopez-Perez, I. Güvenç, G. de la Roche, M. Kountouris, T. Quek, and J. Zhang, "Enhanced intercell interference coordination challenges in heterogeneous networks," IEEE Wireless Communications, vol. 18, no. 3, pp. 22-30, June 2011.

[2] A. Tall, Z. Altman, and E. Altman, "Self organizing strategies for enhanced ICIC (eICIC)," CoRR, vol. abs/1401.2369, 2014. [Online]. Available: http://arxiv.org/abs/1401.2369

[3] S. Deb, P. Monogioudis, J. Miernik, and J. Seymour, "Algorithms for enhanced inter-cell interference coordination (eICIC) in LTE HetNets," IEEE/ACM Trans. on Networking, vol. 22, no. 1, pp. 137-150, 2014.

[4] J. Pang, J. Wang, D. Wang, G. Shen, Q. Jiang, and J. Liu, “Optimized time-domain resource partitioning for enhanced inter-cell interference coordination in heterogeneous networks," in IEEE Wireless Communications and Networking Conference (WCNC), April 2012, pp. 1613-1617.

[5] M. Simsek, M. Bennis, and I. Güvenç, "Enhanced intercell interference coordination in HetNets: Single vs. multiflow approach," in IEEE Globecom Workshops (GC Wkshps), Dec 2013, pp. 725-729.

[6] — "Learning based frequency- and time-domain inter-cell interference coordination in HetNets," CoRR, vol. abs/1411.5548, 2014. [Online]. Available: http://arxiv.org/abs/1411.5548

[7] I.-H. Hou and C. S. Chen, "Self-organized resource allocation in LTE systems with weighted proportional fairness," in IEEE International Conference on Communications (ICC), 2012, pp. 5348-5353.

[8] J. O. Neel, "Analysis and Design of Cognitive Radio Networks and Distributed Radio Resource Management Algorithms," Ph.D. dissertation, Virginia Polytechnic Institute and State University, Sep 2006.

[9] C. Singh and C. S. Chen, "Distributed downlink resource allocation in cellular networks through spatial adaptive play," in 25th International Teletraffic Congress (ITC), 2013.

[10] C. S. Chen, K. W. Shum, and C. W. Sung, "Round-robin power control for the weighted sum rate maximisation of wireless networks over multiple interfering links," European Transactions on Telecommunications, vol. 22, no. 8, pp. 458-470, 2011

[11] S. Sesia, I. Toufik, and M. Baker, LTE - The UMTS Long Term Evolution: From Theory to Practice. Wiley, 2009. 